\title{
REVISÃO SISTEMÁTICA: CUTIS MARMORATA TELANGIECTÁSICA CONGÊNITA FRENTE AOS DIAGNÓSTICOS DERMATOLÓGICOS DIFERENCIAIS
}

Marina He Ryi Kim 1; Marina Tricoly Santos'; Rafaela Wojcik Alves de Macedo1; Larissa Gonçalves Leite ${ }^{1}$; Renato Oliveira de Lima²

1. Discente do Centro Universitário São Camilo. SP, Brasil.

2. Docente do Centro Universitário São Camilo. SP, Brasil.

\section{RESUMO:}

A Cutis Marmorata Telangiectásica Congênita (CMTC) consiste em uma doença rara de caráter vascular-cutâneo. É estimado que desde a sua primeira descrição em 1922, sejam presentes cerca de 300 casos na literatura (SHAREEF et al,. 2021). Sua manifestação pode ser puramente cutânea através da presença, desde 0 nascimento, de manchas reticulares eritêmato-violáceas que somem a digitopressão e não respondem ao serem aquecidas, podendo ou não estar associadas à outras anomalias (como malformações vasculares em SNC, glaucoma congênito, hidrocefalia e assimetria de membros). O diagnóstico de CMTC é clínico, dado através das características da lesão cutânea. A fisiopatologia desta doença ainda não é bem esclarecida, porém a hipótese mais aceita é de seja uma doença multifatorial com possível origem genética (BUl et al., 2019). Este artigo tem como finalidade a melhor elucidação do quadro clínico dermatológico de CMTC e seu diagnóstico, comparando com os seus principais diagnósticos dermatológicos diferenciais.

Palavras-chave:"Cutis marmorata telangiectatica congênita "; "CMTC"; "diagnósticos diferenciais dermatológicos";

\section{ABSTRACT:}


Cutis Marmorata Telangiectásica Congênita (CMTC) is a rare vascular-cutaneous disease. Since the first description, in 1992, until the present day there are around 300 cases found in literature (SHAREEF et al,. 2021). The manifestation can be purely cutaneous, by the appearance of a persistent reticulated marbled erythema, which blanches with pressure and does not resolve with heating since the birth, that can be associated with other anomalies (like vascular malformations in central nervous system, congenital glaucoma,hydrocephalus and limber asymmetry). The diagnosis of CMTC is clinical, given by the evaluation of the cutaneous alteration itself. The pathophysiology of this disease is not well known, the most recent and accepted theory is given by a multifactorial disease with genetic origins (BUl et al., 2019). The goal of this article is to better elucidate the clinical aspects of the cutaneous lesion and its diagnosis, comparing it to the main differential dermatological diagnosis.

Key-words: "Cutis marmorata telangiectatica congênita"; "CMTC"; "differential dermatological diagnosis".

\section{INTRODUÇÃO:}

A Cutis Marmorata Telangiectásica Congênita (CMCT) consiste em uma patologia rara que acomete os recém-nascidos, caracterizada por uma má formação vascular. Estima-se na literatura, cerca de 300 casos descritos no mundo todo até os dias atuais (SHAREEF et al,. 2021). Sua fisiopatologia não é muito bem esclarecida ainda. Seu quadro clínico é dado pelo aparecimento de manchas de aspecto rendilhado, de coloração eritemato-violáceas que se acentuam com o frio, podendo ser encontradas em qualquer parte do corpo, com exceção das plantas de pés e palmas das mãos, sendo mais comumente encontradas em membros inferiores e tronco, de modo unilateral e respeitando os limites da linha média. Seu diagnóstico é puramente clínico. Quando apresenta exclusivamente seu acometimento cutâneo, costuma cursar com bom prognóstico, uma vez que na grande maioria dos casos foi relatado sua regressão espontânea com o desenvolvimento da criança.

Por seu diagnóstico ser baseado em aspectos dermatológicos, foi notada uma dificuldade para distinguir a CMTC de seus diagnósticos diferenciais, uma vez que outras patologias apresentam a mesma descrição da lesão cutânea. Com isso, esse 
estudo foi realizado com a finalidade de facilitar seu diagnóstico frente aos seus diferenciais.

\section{METODOLOGIA}

Pesquisou-se pelas palavras chaves "Cutis marmorata telangiectatica congênita" e "differential dermatological diagnosis" nas bases de dados "BIREME", "Scielo" e "PubMed". Foram encontrados 80 artigos. Desses foram excluídos 9 artigos por duplicação, 10 artigos por serem revisão sistemática, 21 não foram possíveis de acesso na íntegra e 21 que não se encaixavam ao tema. Restaram 19 trabalhos e baseados neles foi elaborada a presente revisão sistemática.

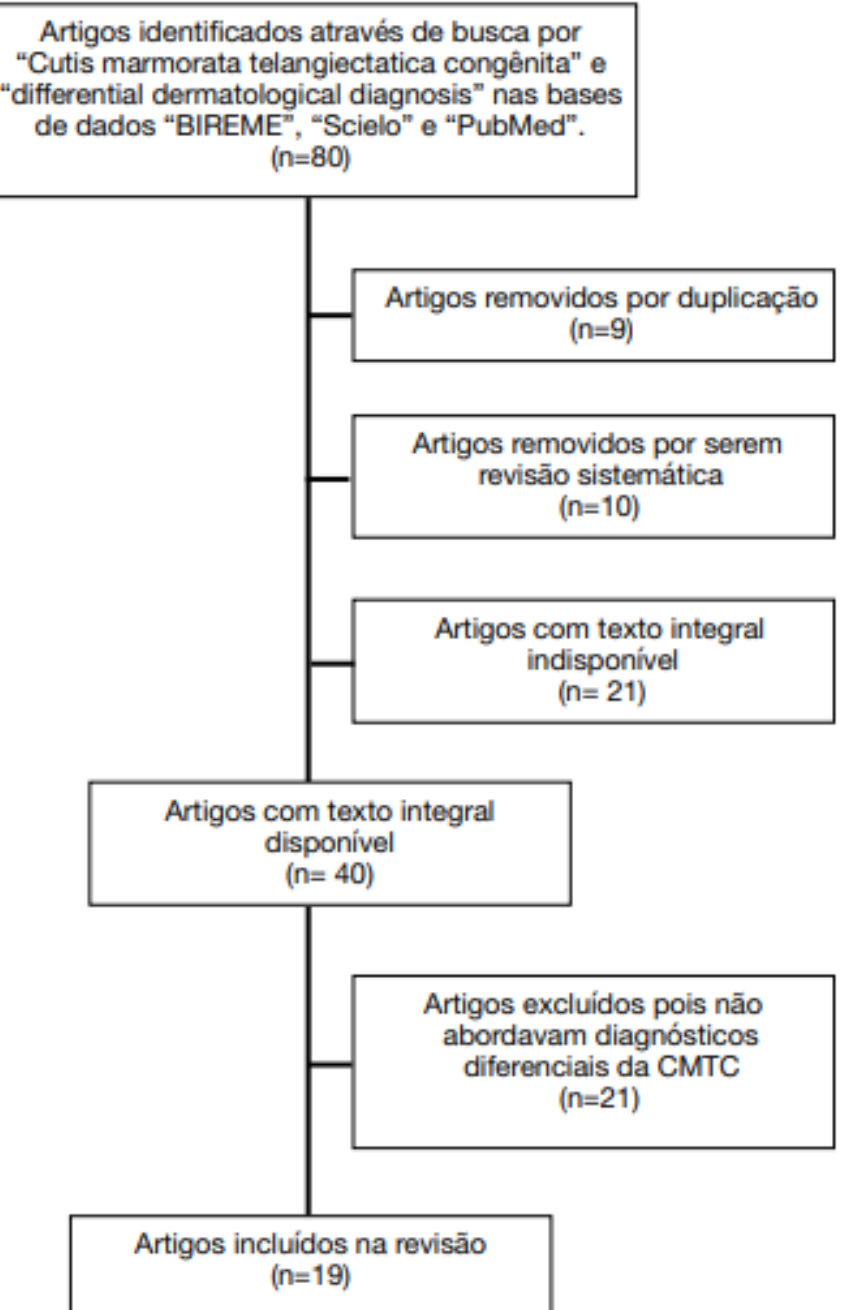




\section{RESULTADOS}

Da seleção de artigos realizada, foram mencionadas como diagnóstico diferencial da CMTC, as seguintes doenças:

\section{Cutis Marmorata Fisiológica}

A cútis marmorata fisiológica ocorre em $\mathrm{RN}$, devido a dilatação dos capilares quando expostos ao frio. Caracteriza-se clinicamente pela presença de um reticulado eritemato-violácea no tronco e extremidades que desaparecem com o aquecimento da região acometida. Esta situação não tem significado patológico.

\section{Hemangioma Infantil}

O hemangioma infantil consiste no tumor vascular benigno mais comum da infância. Sua manifestação não é comum ao nascimento, mas geralmente, sua apresentação ocorre nos primeiros meses de vida, sendo caracterizada como uma mancha ou tumoração de coloração vermelho-arroxeada, principalmente em face e pescoço. Sua evolução natural é bem conhecida, a lesão tende a crescer durante o primeiro ano de vida e após esse período, tende a regredir espontaneamente ao longo da vida. Seu aparecimento é mais frequente em RN de mães que passaram por algum tipo de procedimento invasivo durante a gestação, $\mathrm{RN}$ prematuros e de baixo peso. Com isso, podemos diferenciar da CMTC pela história natural da doença, pois o hemangioma infantil não é comum ao nascimento, tem um aumento na lesão durante o primeiro ano de vida e segue em involução.

\section{Malformações Capilares}

Malformações capilares, também conhecidas como manchas vinho do porto, é uma das malformações vasculares mais comuns. São lesões presentes ao nascimento, mais comuns na cabeça e pescoço, apesar de poderem estar localizadas em qualquer parte do corpo. Podem ser únicas ou múltiplas e variar significativamente de tamanho. Tendem a ser rosadas ao nascimento, e tornam-se mais vermelhas ou violáceas com o tempo. Podem também ser confluentes ou reticuladas. Com o tempo podem se tornar hiperqueratósicas.

\section{Síndrome de Sturge- Weber}


A Sd. Sturge Weber consiste em uma doença rara e congênita, classificada dentro do grupo das facomatoses. É marcada pelo acometimento neurológico, ocular e cutâneo devido a proliferação anormal de capilares. Dentro das suas manifestações cutâneas temos o angioma facial e a mancha vinho do porto, sendo que a primeira é classificada como um tumor vascular benigno, enquanto a segunda consiste em uma mancha de coloração rósea decorrente de uma malformação vascular de capilares, com distribuição que segue os dermátomos, especificamente os ramos sensitivos do trigêmeo. Ambas estão presentes no nascimento e têm crescimento proporcional ao desenvolvimento da criança. Diferentemente da CMTC, o local de maior acometimento das lesões cutâneas na Sd. Sturge Weber é na região crânio-facial.

\section{Síndrome de Klippel- Trenaunay (SKT)}

A síndrome de Klippel-Trenaunay é uma doença rara, caracterizada tríade: manchas vinho do porto (também conhecido como hemangioma plano), malformações venosas ou veias varicosas e hipertrofia óssea e/ou tecidual, cuja fisiopatologia ainda não é muito bem esclarecida. Na maioria dos casos, as manchas e veias varicosas podem estar presentes desde o nascimento e proeminentes até a adolescência, sendo que em $70 \%$ dos casos também estão associadas à alterações linfáticas, que podem resultar em linfedema, linforreia e aumentar a susceptibilidade à celulite. Geralmente acomete membros inferiores, mas pode acometer o tronco. Seu diagnóstico é clínico. Apesar das manchas da sd Klippel-Trenaunay apresentarem caráter vascular como as da CMTC, e da topografia mais acometida ser a mesma, a SKT se diferencia da CMTC pela evolução natural da doença, uma vez que a mancha da SKT tende a crescer conforme o desenvolvimento e não costuma a involuir espontaneamente. Além disso, ainda temos os outros achados como varizes e hipertrofia óssea e/ou de tecidos moles.

\section{Hamartoma de Músculo Liso}

O hamartoma de músculo liso consiste em uma lesão cutânea congênita rara, caracterizada pela proliferação de de células musculares lisas na derme reticular. Em alguns cenários, a lesão cutânea pode imitar a lesão da CMTC como em um caso relatado (Nicole Knöpfel et al., 2020), onde um menino de 2 meses de idade 
apresentou uma lesão livedóide de coloração eritemato-violácea esbranquiçada,com áreas de atrofia e bem delimitada em membro inferior direito. Seu diagnóstico é dado pela biópsia da lesão cutânea, no qual é evidenciado uma epiderme normal, pequenos capilares e vênulas presentes e muitas bandas bem definidas de músculo liso na derme com diferentes direções, que podem se estender até o subcutâneo superficial, além de positivar para os marcadores imunohistológicos como actina de músculo liso (SMA) e calponina.

\section{Eritema ab igne}

O eritema ab igne (EAl) ou eritema calórico é uma dermatose rara, dependente de calor. Sua manifestação é dada pelo aparecimento de um eritema reticulado com hiperpigmentação e descamação, podendo ter áreas de atrofia epidérmica e telangiectasias. Costuma acometer regiões de maior exposição ao calor, sendo o principal sítio a região lombossacral, geralmente associada ao uso de bolsas de água quente e termoterapia para analgesia de dores crônicas. Seu diagnóstico é facilmente dado pela morfologia da lesão juntamente com a história de exposição excessiva ao calor uma vez que as lesões aparecem com o tempo pois estão relacionadas com a exposição ao calor e não estão presentes desde o nascimento.

\section{Síndrome Antifosfolipide (SAF)}

A Sd. antifosfolipide consiste em uma condição adquirida e sistêmica, de caráter autoimune, cuja apresentação é dada por trombose arterial e/ou venosa, abortos espontâneos recorrentes, trombocitopenia, acompanhada de títulos elevados de anticorpos antifosfolípides (AAF): anticoagulante lúpico e/ou anticardiolipina. É mais incidente em mulheres adultas jovens, e pode ser primária ou secundária a alguma outra patologia como o lúpus eritematoso sistêmico. A maioria dos pacientes portadores desta síndrome apresentam alterações cutâneas, sendo que cerca de $41 \%$ deles apresentam alterações cutâneas como sua primeira manifestação (Jesus Rodrigues Samaria, et. al.) sendo essa caracterizada por lesões vásculo-cutâneas rendilhadas de coloração purpúrica que podem estar, ou não, associadas com úlceras. Seu diagnóstico é dado por critérios já pré-estabelecidos pelo Consenso Internacional de critérios preliminares para a classificação da Sd. antifosfolipide. Apesar da epidemiologia e das manifestações extra-cutâneas serem muito 
divergentes às da CMTC, a SAF foi mencionada devido a existência de casos de SAF secundária ao lúpus neonatal diagnosticada em crianças com menos de 1 ano de idade.

\section{Síndrome de Adams-Oliver}

A Síndrome de Adams-Oliver é caracterizada como defeitos no couro cabeludo (aplasia de cutis), defeitos terminais transversais de membros e outra anomalia sistêmica ou física.

Incidência de 1/10000 nascidos vivos, distribuição igual entre os sexos. A maioria dos casos é autossômica dominante.

O couro cabeludo é a região mais afetada, além da atrofia de pele com veias tortuosas e dilatadas, podendo essa lesão gerar atrofia local e chegar até as meninges.

Associada pode apresentar no restante do corpo sindactilia, polidactilia, aplasia de cutis, hiperpigmentação e hemangiomas. Manifestações sistêmicas são: alterações cardíacas, hidronefrose, criptorquidismo, alterações neurológicas.

Diagnóstico é puramente clínico. As lesões tendem a sumir até 3 anos de vida, mas depende das condições associadas e, geralmente, os óbitos ocorrem por infecções respiratórias. Diferentemente da CMTC o local mais acometido é o couro cabeludo, além de estar associada a sindactilia, polidactilia e aplasia de cutis.

\section{Síndrome de Cutis Marmorata Macrocefálica}

Uma síndrome de crescimento excessivo reconhecida por Moore et al. [1997] e Clayton-Smith et al. [1997]. Essa síndrome é caracterizada por macrocefalia, altura acima do percentil 90, e mais dois achados como cutis marmorata, angioma, sindactilia e assimetria. [Franceschini et al., 2000]. Quarenta e cinco pacientes foram reportados com essa síndrome até hoje.

\section{Lúpus Neonatal}

O lúpus neonatal é definido pelo desenvolvimento de lesões cutâneas, defeitos cardíacos de condução, ou ambos ocorrendo em recém nascidos de mãe com Lúpus eritematoso sistêmico clínico ou subclinico ou síndrome de Sjogren. Anti-Ro e anti-I, e excepcionalmente anti-U, estão presentes tanto em mãe como criança. As 
lesões cutâneas consistem usualmente em placas eritematosas edemaciadas presentes em cabeça e pescoço.

\section{Síndrome de Bockenheimer}

É uma malformação hamartomatosa progressiva que acomete veias profundas, com estabelecimento gradual na infância e dilatações venosas irregulares dolorosas que costumam afetar apenas 1 membro.

\section{DISCUSSÃO}

A CMCT é uma patologia rara que acomete recém-nascidos, caracterizada por uma má formação vascular que visualmente se assemelha ao mármore, o que levou ao nome da doença.

Há aproximadamente 300 casos descritos no mundo até os dias atuais (SHAREEF et al,. 2021), sendo o primeiro deles descrito em 1922, pelo pediatra holandês, Cato van Lohuizen. Existem divergências na literatura referente a sua prevalência conforme os gêneros. Sua fisiopatologia não é muito bem esclarecida, porém estudos recentes sugerem uma possível origem genética (BUl et al., 2019), e pode estar associada a consanguinidade pela mutação do gene ARL6IP6 (Mon R.A et. $\mathrm{al}$, ).

Seu quadro clínico é caracterizado pelo aparecimento de manchas rendilhadas, eritemato-violáceas que se tornam mais visíveis com o frio, podendo ser encontradas em qualquer parte do corpo, com exceção das plantas de pés e palmas das mãos. São mais comumente encontradas em membros inferiores e tronco, de modo unilateral e respeitando os limites da linha média. Além disso, existem outras anormalidades associadas descritas na literatura, sendo a mais comum a assimetria de membros, além de malformações esqueléticas e outras alterações vasculares em diferentes órgãos tendo seus achados clínicos conforme o órgão afetado.

Seu diagnóstico é puramente clínico, pelo aspecto da lesão cutânea, não sendo necessária a biópsia da lesão, uma vez que não há nenhum achado específico da CMTC na mesma. Contudo, devido a possibilidade do acometimento de vasos sanguíneos de órgãos nobres, após o diagnóstico de CMTC, é realizada uma 
investigação com exames complementares como método de rastreio para outras anormalidades vasculares. Os exames mais solicitados são a fundoscopia, o USG transfontanela, o USG de abdome total e o Ecocardiograma.

Devido ao seu diagnóstico ser clínico, muitas vezes é difícil diagnosticá-la. Dessa forma elaboramos uma tabela com os principais achados de diagnósticos diferenciais e suas diferenças em relação à CMTC

\begin{tabular}{|c|l|}
\hline \multicolumn{1}{|c|}{ DIAG. DIFERENCIAIS } & \multicolumn{1}{|c|}{ DIFERENÇA QUANTO À CMTC } \\
\hline Cutis Marmorata Fisiológica & $\begin{array}{l}\text { Desaparecimento da mancha quando } \\
\text { exposta a temperaturas mais quentes. }\end{array}$ \\
\hline Hemangioma Infantil & $\begin{array}{l}\text { Não é comum ao nascimento. } \\
\text { Aumento na lesão durante o primeiro ano de } \\
\text { vida } \\
\text { e tendem a involuir com o tempo após esse } \\
\text { período. }\end{array}$ \\
\hline Malformações & $\begin{array}{l}\text { São lesões presentes ao nascimento, mais } \\
\text { comuns na cabeça e pescoço, apesar de } \\
\text { poderem estar localizadas em qualquer parte } \\
\text { do corpo. Podem ser únicas ou múltiplas e } \\
\text { variar significativamente de tamanho. } \\
\text { Tendem a ser rosadas ao nascimento, e } \\
\text { tornam-se mais vermelhas ou violáceas com } \\
\text { o tempo. } \\
\text { Podem também serem confluentes ou } \\
\text { reticuladas. Com o tempo podem se tornar } \\
\text { hiperqueratosicas. }\end{array}$ \\
\hline Síndrome de Sturge-Weber & $\begin{array}{l}\text { Local de maior acometimento das lesões } \\
\text { cutâneas é na região crânio-facial. } \\
\text { Algumas lesões podem ser malignas e }\end{array}$ \\
\hline
\end{tabular}




\begin{tabular}{|c|c|}
\hline & cursar com um prognóstico ruim. \\
\hline Síndrome Klippel- Trenaunay & $\begin{array}{l}\text { A mancha tende a crescer conforme o } \\
\text { desenvolvimento e não costuma a involuir } \\
\text { espontaneamente. } \\
\text { Pode estar associada a varizes e hipertrofia } \\
\text { óssea e/ou de tecidos moles. }\end{array}$ \\
\hline Hamartoma de Músculo Liso & $\begin{array}{l}\text { Diagnóstico é pela biópsia que visualiza } \\
\text { muitas bandas bem definidas de músculo liso } \\
\text { na derme com diferentes direções, que } \\
\text { podem se estender até o subcutâneo } \\
\text { superficial. } \\
\text { Positiva os marcadores imunohistológicos } \\
\text { como actina de músculo liso (SMA) e } \\
\text { calponina. }\end{array}$ \\
\hline Eritema ab igne & $\begin{array}{l}\text { Lesão depende e exposição prolongada ao } \\
\text { calor e pode apresentar atrofia local. } \\
\text { O principal sítio de acometimento é a região } \\
\text { lombossacral. } \\
\text { As lesões cutâneas aparecem com o tempo e } \\
\text { não estão presentes desde o nascimento. }\end{array}$ \\
\hline $\begin{array}{l}\text { Síndrome antifosfolípide } \\
\text { (SAF) }\end{array}$ & $\begin{array}{l}\text { Acomete mulheres jovens. } \\
\text { Pode ser secundária a outras patologias. } \\
\text { Apresenta manifestação extracutânea } \\
\text { associada. }\end{array}$ \\
\hline Síndrome de Adams-Oliver & $\begin{array}{l}\text { O local mais acometido é o couro cabeludo. } \\
\text { Pode estar associada a sindactilia, polidactilia } \\
\text { e aplasia de cutis. } \\
\text { Suas lesões em couro cabeludo costumam } \\
\text { cursar com atrofia local. }\end{array}$ \\
\hline
\end{tabular}




\begin{tabular}{|c|l|}
\hline $\begin{array}{c}\text { Síndrome de Cutis Marmorata } \\
\text { Macrocefálica (M-CMTC) }\end{array}$ & $\begin{array}{l}\text { Essa síndrome é caracterizada por } \\
\text { macrocefalia, altura acima do percentil 90, e } \\
\text { mais dois achados como cutis marmorata, } \\
\text { angioma, sindactilia e assimetria. }\end{array}$ \\
\hline Lúpus Neonatal & $\begin{array}{l}\text { O lúpus neonatal é definido pelo } \\
\text { desenvolvimento de lesões cutâneas e/ou } \\
\text { defeitos cardíacos de condução, ocorrendo } \\
\text { em recém nascidos de mãe com Lúpus } \\
\text { eritematoso sistêmico clínico, subclinico ou } \\
\text { síndrome de Sjogren. Anti-Ro e anti-I, e } \\
\text { excepcionalmente anti-U, estão presentes } \\
\text { tanto em mãe como criança. }\end{array}$ \\
\hline Síndrome de Bockenheimer & $\begin{array}{l}\text { Estabelecimento gradual na infância, não é } \\
\text { comum ao nascimento e costuma afetar } \\
\text { apenas 1 membro. }\end{array}$ \\
\hline
\end{tabular}

Tabela 1: diagnósticos dermatológicos diferenciais de CMTC e seus principais achados.

Quando apresenta exclusivamente seu acometimento cutâneo, a CMTC costuma cursar com bom prognóstico, uma vez que na grande maioria dos casos foi relatado sua regressão espontânea com o desenvolvimento da criança, o que também difere a mesma com alguns de seus diferenciais.

\section{CONCLUSÃO}

Os profissionais de saúde encontram dificuldade em fechar o diagnóstico da Cutis Marmorata Telangiectásica Congênita (CMTC) por ser uma doença rara, com poucos casos descritos na literatura e também por suas semelhanças com os diagnósticos diferenciais.A importância desse estudo é disseminar o conhecimento atual sobre o diagnóstico da CMTC frente aos outros diagnósticos diferenciais, para que quando os profissionais se depararem com esse contexto, estejam mais orientados para realizar o seu diagnóstico e tratá-la corretamente. Por ser um 
diagnóstico puramente clínico, é necessário que se conheça bem as características da lesão: manchas rendilhadas, eritemato-violáceas que se tornam mais visíveis com o frio, podendo ser encontradas em qualquer parte do corpo, com exceção das plantas de pés e palmas das mãos e que não somem com o aumento de temperatura no local. São mais comumente encontradas em membros inferiores e tronco, de modo unilateral e respeitando os limites da linha média. Além disso, seu diagnóstico de forma correta é fundamental para o rastreio e manejo de outras possíveis malformações vasculares que possam repercutir na qualidade de vida do paciente, uma vez que na CMTC é possível encontrarmos alterações na vascularização de cérebro, coração e rins, o que também ressalta a importância do diagnóstico de forma precoce.

\section{REFERÊNCIAS:}

Blanc J, Bodak N, Saada V, Zavaro A, Bagot M, Petit A, Bourrat E. Polymorphisme et diagnostics différentiels du lupus érythémateux néonatal [Polymorphism and differential diagnosis of neonatal lupus erythematosus]. Ann Dermatol Venereol. 2020 Apr;147(4):258-264. French. doi: 10.1016/j.annder.2019.09.613. Epub 2020 Mar 12. PMID: 32171550.

Carrascosa JM, Ribera M, Bielsa I, Coroleu W, Ferrándiz C. Cutis marmorata telangiectatica congenita or neonatal lupus? Pediatr Dermatol. 1996 MayJun;13(3):230-2. doi: 10.1111/j.1525-1470.1996.tb01209.x. PMID: 8806125.

Dupont C. Cutis marmorata telangiectatica congenita (Van Lohuizen's syndrome). Br J Dermatol. 1977 Oct;97(4):437-9. doi: 10.1111/j.1365-2133.1977.tb14254.x. PMID: 588453.

Ergon EY, Çelik K, Çolak R, Özdemir SA, Olukman Ö, Kundak S, Çalkavur Ş. A rare vascular lesion of newborn: cutis marmorata telangiectatica congenital. Turk Pediatri Ars. 2019 Jul 11;54(2):133-136. doi: 10.5152/TurkPediatriArs.2018.5557. PMID: 31384150; PMCID: PMC6666354.

Fayol L, Garcia P, Denis D, Philip N, Simeoni U. Adams-Oliver syndrome associated with cutis marmorata telangiectatica congenita and congenital cataract: a case report. Am J Perinatol. 2006 Apr;23(3):197-200. doi: 10.1055/s2006-934099. Epub 2006 Mar 29. PMID: 16586236. 
Frantz, J.A.M., Lehmkuhl, R.L., Leitis, L.H., Uliano, V.G. and Siementcoski, G.A. (2015), Adams-Oliver Syndrome: A Case Report. Pediatr Dermatol, 32: 383-385. https://doi.org/10.1111/pde.12423

Imafuku S, Tashiro A, Furue M, Nakayama J. Cutis marmorata telangiectatica congenita manifesting as port-wine stain at birth. J Dermatol. 2008 Jul;35(7):471 2. doi: 10.1111/j.1346-8138.2008.00505.x. PMID: 18705838.

J. del Boz González, M.M. Serrano Martín, A. Vera Casaño, Cutis marmorata telangiectásica congénita. Revisión de 33 casos, Anales de Pediatría,Vol. 69, Issue 6, 2008; 557-564, ISSN 16954033,https://doi.org/10.1016/S1695-4033(08)75239-1.

Jafse M. Carrascosa M.D., Miquel Ribera M.D., Isabel Bielsa M.D., Wenceslao Coroleu M.D., Carlos Ferrándiz M.D. Cutis Marmorata Telangiectatica Congenita or Neonatal Lupus? doi: 10.1111/j.1525-1470.1996.tb01209.x

Karlsson JP, Telang GH, Tunnessen WW Jr. Picture of the month. Cutis marmorata telangiectatica congenita. Arch Pediatr Adolesc Med. 1997 Sep;151(9):949-50. doi: 10.1001/archpedi.1997.02170460087016. PMID: 9308876.

Knöpfel N, Butzmann CM, Büchner A, Theiler M, Fraitag S, Weibel L. Cutis marmorata telangiectatica congenita-like lesion with fibrotic appearance. Pediatr Dermatol. 2020 Jan;37(1):204-206. doi: 10.1111/pde.13958. PMID: 31997445.

Liu W, Ma DL, Vano-Galvan S. Image Gallery: Atrophic cutis marmorata telangiectatica congenita. Br J Dermatol. 2019 Aug;181(2):e32. doi: 10.1111/bjd.17846. Epub 2019 Jun 26. PMID: 31243761.

Mégarbané A, Haddad J, Lyonnet S, Clayton-Smith J. Child with overgrowth, pigmentary streaks, polydactyly, and intestinal lymphangiectasia: macrocephalycutis marmorata telangiectatica congenita syndrome or new disorder? Am J Med Genet A. 2003 Jan 15;116A(2):184-7. doi: 10.1002/ajmg.a.10705. PMID: 12494441.

Milligan A, Graham-Brown RAC. Erythema ab igne affecting the palms. Clin Exp Dermatol. 1989;14:168-9.

Mon R. A, Mozurkewich E, Treadwell M, C, Berman D, R: Cutis Marmorata Telangiectatica Congenita Presenting as a Fetal Hemothorax. Fetal Diagn Ther 2019;45:281-284. doi: 10.1159/000488487. 
Nyberg RH, Uotila J, Kirkinen P, Rosendahl H. Macrocephaly-cutis marmorata telangiectatica congenita syndrome--prenatal signs in ultrasonography. Prenat Diagn. 2005 Feb;25(2):129-32. doi: 10.1002/pd.1081. PMID: 15712320.

Poliak N, Rainey A. Concurrent Sturge-Weber syndrome, facial infantile hemangioma, and cutis marmorata telangiectatica congenita. Cutis. 2017 Oct;100(4):252-254. PMID: 29136059.

Redondo P, Aguado L, Martínez-Cuesta A. Diagnosis and management of extensive vascular malformations of the lower limb: part I. Clinical diagnosis. J Am Acad Dermatol. 2011 Nov;65(5):893-906; quiz 907-8. doi: 10.1016/j.jaad.2010.12.047. PMID: 22000870.

Reticular rash in a neonate. J Paediatr Child Health. 2018 Feb;54(2):208. doi: 10.1111/jpc.2_13782. PMID: 29417663.

Rupprecht R, Hundeiker M. Cutis marmorata teleangiectatica congenita. Wichtige Aspekte für die dermatologische Praxis [Cutis marmorata teleangiectatica congenita. Important aspects for dermatologic practice]. Hautarzt. 1997 Jan;48(1):21-5. German. doi: 10.1007/s001050050541. PMID: 9132383.

Santamaria, Jesus Rodriguez et al. Síndrome antifosfolípide. Anais Brasileiros de Dermatologia [online]. 2005, v. 80, n. 3 [Acessado 10 Julho 2021] , pp. 225-239. Disponível em: <https://doi.org/10.1590/S0365-05962005000300002>. Epub 03 Nov 2005. ISSN 1806-4841. https://doi.org/10.1590/S0365-05962005000300002.

Torrelo A, Zambrano A, Happle R. Large aberrant Mongolian spots coexisting with cutis marmorata telangiectatica congenita (phacomatosis pigmentovascularis type $\mathrm{V}$ or phacomatosis cesiomarmorata). J Eur Acad Dermatol Venereol. 2006 Mar;20(3):308-10. doi: 10.1111/j.1468-3083.2006.01395.x. PMID: 16503893.

Trevisan F, Cunha PR, Pinto CA, Cattete FG. Cutaneous neonatal lupus with cutis marmorata telangiectatica congenita-like lesions. An Bras Dermatol. 2013 MayJun;88(3):428-31. doi: 10.1590/abd1806-4841.20131832. PMID: 23793203; PMCID: PMC3754378.

Watson T, Martinez E, Crabbe D, Chetcuti P, Kraft JK. Renal lymphangiomatosis, interrupted IVC with persistent primitive hepatic venous plexus and multiple anomalous venous channels: parts of an overlap syndrome? Pediatr Radiol. 2012 Feb;42(2):253-6. doi: 10.1007/s00247-011-2184-2. Epub 2011 Jul 20. PMID: 21773796. 
Weber, Magda Blessmann et al. Eritema ab igne: relato de um caso. Anais Brasileiros de Dermatologia [online]. 2005, v. 80, n. 2 [Acessado 10 Julho 2021] , pp. 187-188. Disponível em: <https://doi.org/10.1590/S0365-

05962005000200010>. Epub 13 Jun 2005. ISSN 1806-4841. https://doi.org/10.1590/S0365-05962005000200010 